\title{
PERFIL DOS DESLOCAMENTOS PENDULARES POR MOTIVO DE ESTUDO NO ESTADO DO CEARÁ EM 2010
}

\author{
Tallita Soares Justino ${ }^{2}$ \\ Italo Eduardo Brito da Costa ${ }^{3}$
}

\section{Resumo}

Diversos estudos sobre pendularidade abordam a complexidade atual da organização do espaço, que cada vez mais ganha-se visibilidade os fluxos diários de pessoas. Nesse sentido, o presente trabalho define deslocamento como o trajeto dos indivíduos que estudam em um município ou país estrangeiro diferente daquele em que reside. O estudo objetiva analisar o perfil dos indivíduos que se deslocam regularmente para outro município ou país estrangeiro em busca de elevação do nível de escolaridade, no Estado do Ceará em 2010. Para tanto, utilizou-se os microdados da amostra do Censo Demográfico 2010. Em que, os resultados indicam uma predominância do de indivíduos sexo feminino com maior grau de escolaridade em relação ao sexo masculino, com participação mais expressiva na faixa de idade de 14 a 28 anos, solteiros, resistentes da zona rural e que cursam o ensino superior de graduação. Além disso, observouse que os fluxos ocorrem principalmente para o município de Fortaleza, secundariamente destaca-se a participação de municípios tais como: sobral, Juazeiro do Norte, Crato e Maracanaú, Quixadá e Limoeiro do Norte.

Palavras-chave: Pendularidade, Ceará, Escolaridade, Estatística Descritiva

\section{Introdução}

Parte-se da compreensão de que a mobilidade pendular refere-se ao processo de deslocamento da população no território, inseridas num determinado contexto e tempo socialmente constituídos, pois está diretamente relacionada com as diferentes trajetórias espaciais da população (JARDIM, 2007).

Nesse sentido o Instituto Brasileiro de Geografia e Estatística (IBGE) (2007) denomina como deslocamento pendular o fluxo de pessoas entre regiões ou localidade, motivadas, muitas vezes, por questão de necessidade, de sobrevivência ou pela busca de novas oportunidades, sejam elas de emprego, melhor qualidade de vida ou até elevar o nível de escolaridade.

Conforme Ântico (2005) e Jardim (2001), os movimentos urbanos cotidianos apesar de serem processos cujos estudos são incipientes, cada vez mais, ganham força na atualidade

\footnotetext{
${ }^{1}$ Registro meu sincero agradecimento ao Professor Ricardo Ojima por importantes contribuições nesse trabalho.

${ }^{2}$ Mestranda em Demografia pelo Programa de Pós-Graduação em Demografia da Universidade Federal do Rio Grande do Norte (UFRN), Rio Grande do Norte/Brasil. E-mail: tallitasj_21@ @otmail.com

${ }^{3}$ Graduando em Ciências Atuariais pela Universidade Federal do Rio Grande do Norte (UFRN), Rio Grande do Norte/Brasil. E-mail: itinhoeduar@gmail.com
} 
tendo como principais fatores motivadores trabalho ou estudo. Tem-se que, tais deslocamentos estão associados aos grandes aglomerados urbanos, devido ao fato de que eles recebem fluxos pendulares substanciais seja no perímetro metropolitano e especialmente aqueles relacionados aos deslocamentos intermunicipais.

Dessa forma, para utilização e interpretação dos fluxos pendulares no Estado do Ceará em 2010, compreende-se que a mobilidade pendular de estudantes é um tipo de deslocamento espacial motivado pelo acesso ao local de estudo. Considera-se ainda, que os movimentos populacionais regulares, não necessariamente diários, sem implicação de mudança de residência. Nesse sentido, busca-se, aqui, destacar, dentre inúmeros motivos, o acesso a escolaridade em direção a um Município ou País Estrangeiro distinto daquele de residência.

Sabe-se que os investimentos implementados pelo Governo Federal a fim de reverter a precária realidade da educação no Brasil não têm sido suficientes a ponto de oferecer uma estrutura capaz de corresponder as carências presentes no ensino público na grande maioria do território nacional, em todos os níveis de formação. Esse quadro tende a tornar-se mais crítico em determinadas regiões brasileiras, nas quais, os reflexos dos investimentos de recursos públicos destinados à educação, menos aparecem (MOREIRA et al, 2013).

Num estudo empreendido por Cunha e Jakob (2010), para a área metropolitana de Campinas, indica que a mobilidade pendular é um tipo de deslocamento que pode favorecer o indivíduo, evidenciando o lado vantajoso de tal movimento, à medida que que as pessoas o tem como uma oportunidade de acesso ao estudo em outros municípios ou país estrangeiro.

Poucos são os trabalhos que tem como recorte territorial o Estado do Ceará para analisar o deslocamento pendular por motivo de escolaridade. E os trabalhos até o momento encontrados, a maioria, têm como foco apenas no ensino superior.

Uma exceção são as análises de Ojima; Silva; Pereira (2007) mostrando por meio de uma distribuição dos municípios brasileiros entre 1980 e 2000, por Unidade da Federação e segundo a proporção de movimentos pendulares sobre a população ocupada, que de modo geral há um aumento dos municípios que se encontram em níveis de pendularidade mais elevadas de 1980 para 2000. Analisando o caso do Ceará, observou-se que houve um aumento significativo na proporção de municípios que possuíam entre $20 \%$ e $30 \%$ de pendularidade, passando de menos de $1 \%$ para $2 \%$, e os de mais de $40 \%$ passou de 0 para mais de $2 \%$ dos municípios desta UF entre 1980 e 2000.

De modo geral, há uma série de pesquisas que analisam os movimentos pendulares com foco, em maior proporção, nas aglomerações urbanas brasileiras ou entre Regiões 
Metropolitanas (ÂNTICO, 2005; PESSOA, 2005; BARCELLOS; JARDIM, 2011; BRITO, 2014; LAMEIRA, 2014), mas no que se refere a análise dos movimentos pendulares do Ceará não é muito explorado. Elaborado a partir de uma revisão sistemática da literatura, o presente artigo tem o objetivo de analisar algumas das características (sexo, idade, curso que frequenta, cor ou raça, situação domiciliar, estado civil) dos indivíduos, do Estado do Ceará em 2010, que se deslocam regularmente para outro município ou país estrangeiro em busca de elevar o grau de escolaridade.

\section{Metodologia}

A área de estudo refere-se ao Estado do Ceará, cuja localização geográfica pode ser vista no mapa 1. Segundo o senso de 2010 O referido Estado ocupa uma área de 148.886,308 $\mathrm{km}^{2}$ e uma população de 8.452.381 habitantes, distribuídos em 184 municípios. A cidade mais populosa é a capital, Fortaleza, sede da Região Metropolitana de Fortaleza (RMF), com 2.452.185 habitantes. A densidade demográfica do Estado é de 56,76 habitantes por $\mathrm{Km}^{2}$ (IBGE, 2010).

Figura 1: localização Geográfica do Estado do Ceará.

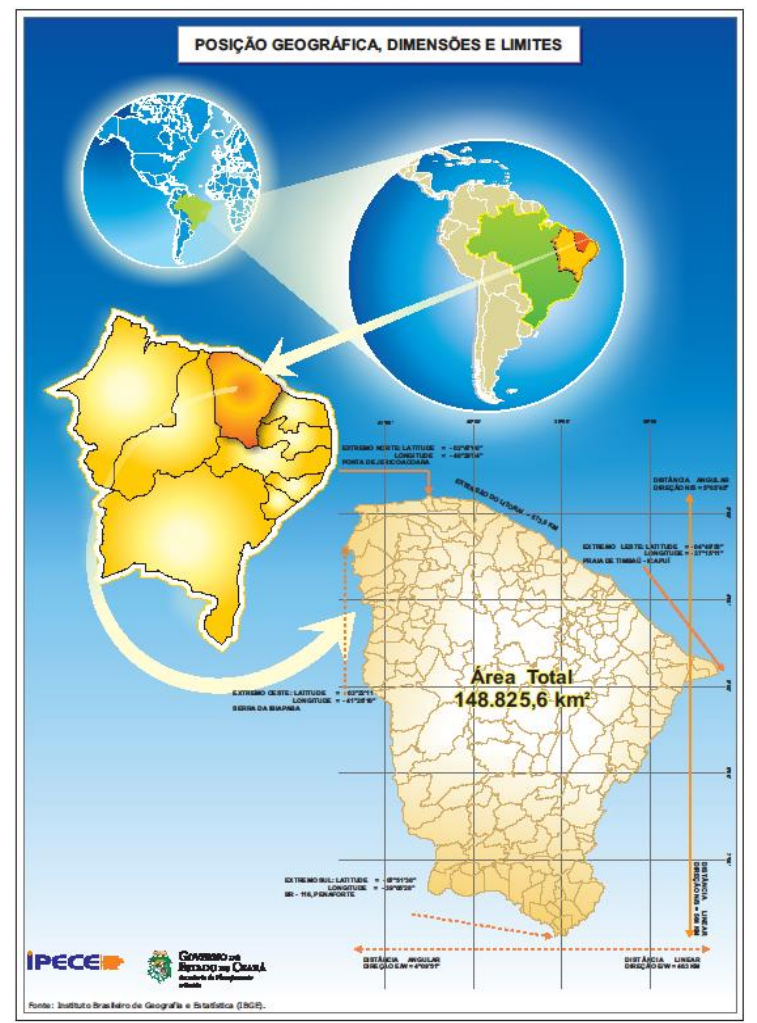

Fonte: Instituto de Pesquisa e Estratégia Econômica do Ceará 
Para traçar o perfil dos indivíduos que pendulam utilizou-se como principal fonte de dados os microdados de Pessoa do Censo Demográfico de 2010 do Instituto Brasileiro de Geografia e Estatística - IBGE, e aplicou-se o método de estatística descritiva através do uso do software SPSS.

Reconhecendo que, essa análise só foi possível porque ouve uma modificação na variável de deslocamento, onde, partir do Censo de 2010 passa a se captar separadamente as informações de movimentos pendulares por motivos de trabalho e motivo estudo.

A análise foi realizada com base na pergunta, segundo a documentação do levantamento censitário de 2010, sobre o local da escola ou creche que o indivíduo frequentava, classificado como: Município de residência, quando a escola ou creche que a pessoa frequentava estava localizada no município em que residia; Outro município, quando a escola ou creche que a pessoa frequentava estava localizada em município diferente daquele em que residia; ou País estrangeiro, quando a escola ou creche que a pessoa frequentava estava localizada em país estrangeiro indivíduos que estavam na situação de pessoa ocupada.

\section{Resultados e discussão}

A partir dos resultados da estatística descritiva identificou-se que, no Estado do Ceará os movimentos pendulares com objetivo de estudo, é mais expressivo entre o sexo feminino $(54,2 \%)$ do que o masculino $(45,8 \%)$.

Já as idades que apresentaram uma maior frequência estão dentro da faixa etária de 14 a $28 \operatorname{anos}(54,9 \%)$ e se autodenominam de cor parda $(57,1 \%)$. Já em relação a situação do domicílio constata-se que uma maioria $(69,5 \%)$ pertencem a zona urbana enquanto uma pequena parcela $(30,5 \%)$ fazem parte da zona rural.

Em decorrência das informações anteriores, pode-se auferir que as relações se dão em sua maioria entre municípios com uma certa urbanização e que há uma variação espacial em termos de oferta de estudo. Nesse sentido a acessibilidade para oportunidade elevação do nível de escolaridade em municípios vizinhos, pode aumenta a as chances de se escolher movimentos pendulares como modo de mobilidade.

No que se refere ao estado civil observou-se uma notável proporção $(71,1 \%)$ de solteiros que realizam o movimento pendular para estudar em um município diferente daquele de residência ou pais estrangeiro, seguido por 13,8\% dos casados, do que nos demais grupos. Essa informação é sem dúvida bastante interessante, pois pode ser um indicativo de que possuir cônjuge restrinja a mobilidade pendular. 
As primeiras constatações indicam que os movimentos pendulares por motivo de escolaridade no Ceará em 2010 são em sua maioria realizados por mulheres, solteiras, entre 14 e 28 anos e que, 37,6\% são ingressantes no nível superior de graduação. Diferentemente dos homens que além de serem uma minoria nesse processo, a maior parcela, 33,9\%, ocupam níveis escolares de baixa instrução, isto é, regular do ensino fundamental.

Não obstante, a literatura brasileira já antecede a maior escolaridade das mulheres em relação aos homens no país. Piçango (2016) evidencia uma menor presença dos jovens homens na escolarização ou o seu pior desempenho e maior presença no mercado de trabalho, frente ao aumento da presença feminina na escolarização e no mercado de trabalho não é apenas um fenômeno que ocorre apenas no Brasil, mas é de caráter internacional. Baylão e Schettino (2014) afirmam que "as mulheres têm ganhado espaço e vem mostrando que podem ocupar qualquer cargo com autoridade".

Possivelmente, por a mulher ser interpretada como limitada, acaba tendo menos oportunidades, no que diz respeito a inserção e, principalmente no que refere-se a menores remuneração no mercado de trabalho, nesse sentido a elevação do nível de instrução torna-se um importante mecanismo para uma melhor aceitação da participação feminina na força de trabalho (ANKER, 2004).

As análises anteriores corroboram com o estudo realizado pelo Instituto de Pesquisa e Estratégia Econômica do Ceará - IPECE (2013), denotando que no Ceará de 2001 a 2011, embora a discriminação entre sexo ainda seja presente em alguns setores econômicos, as mulheres têm mais anos de estudos, maior escolaridade e vêm conquistando aos pouco seu espaço no mercado de trabalho, e por sua vez diminuindo a diferença salarial em relação aos homens.

Analisando o fenômeno da pendularidade na perspectiva do local de partida e do direcionamento do fluxo, constatou-se um maior percentual de total de $11,1 \%$ dos estudantes que que deslocam-se para estudar em fortaleza, dentre os quais $37 \%$ são residentes de Caucaia. Os resultados refletem as ações realizadas pela Lei de Diretrizes e Bases da Educação - LDB (BRASIL, 1996) aumentando o número de instituições de ensino, cursos e vagas no país, além de permitir alternativas de ordem organizacional e curricular. Assim como o Plano Nacional de Educação - PNE (BRASIL, 2001) para o decênio 2001-2010 que promoveu uma política direcionada para a expansão da educação superior como estratégia de desenvolvimento nacional. Em decorrência dessas medidas 
Mora Caucaia e vai para fortaleza $37 \%$ constatou-se que houve uma ampliação do número de vagas em instituições de ensino superior em ritmo mais acelerado que o crescimento populacional, proporcionando maior acesso da população a esse nível de ensino (...) (FUSCO; OJIMA, 2016).

Dessa forma, fica evidente que as políticas voltadas para a ampliação do número de instituições de ensino têm permitido aos indivíduos, que buscam elevar o nível de escolaridade, percorrerem distâncias cada vez menores. Sem a necessidade de fixar residência realizam percursos regulares entre a residência e a instituição de ensino em municípios diferentes, intensificando o deslocamento pendular.

\section{Conclusão}

A presente pesquisa tratou-se de identificar as algumas características dos indivíduos que se deslocam regularmente para outro município ou país estrangeiro em busca de elevar o grau de escolaridade no Estado do Ceará em 2010.

Com base nas informações, resultantes da análise descritiva, conclui-se que, no Estado do Ceará os movimentos pendulares com objetivo de estudo, ocorreu uma concentração entre as mulheres, que ganha destaque em relação a ascensão no nível de escolaridade.

A literatura e as mais diversas pesquisas recentes sobre níveis de escolaridade no Brasil apontam que há um aumento da participação feminina nos últimos anos, e que trata-se de um fenômeno que não possui fronteiras, sendo observado em todo o mundo. Dentre as justificativas que se pode mencionar que para esse processo está a busca pela garantia de melhores salários e formas de trabalho. Baylão e Schettino (2014) afirmam que "trabalhadores mais instruídos ou com mais experiência tendem a ocupar os melhores postos de trabalho pois possuem maior estoque do chamado "capital humano".

Não tão diferente, um estudo realizado pelo Sistema de Pesquisa de Emprego e Desemprego (2011) indica que quanto maior a escolarização do indivíduo, maior é a presença destas pessoas no mercado de trabalho. Outra questão relevante é que os trabalhadores com menor escolarização se depararam com relações mais precárias de trabalho, os de nível superior ocupam postos de trabalho mais formalizados, especialmente no setor público. De modo geral, a escolaridade mais elevada facilitar a obtenção do trabalho. Embora as oportunidades de trabalho e salários não sejam iguais entre os sexos, deve-se destacar que ultimamente tem-se verificado que a presença feminina na força de trabalho é crescente com o aumento da escolaridade. 
Notabiliza-se também que, no Ceará em 2010, esses indivíduos são, em sua maioria, jovens que não possuem nenhum compromisso matrimonial de cor parda e que vivem na zona urbana. Essas características podem indicar uma maior predisposição à pendular, e ainda, que as relações espaciais deixam de ser consideradas apenas entre a zona rural e a zona urbana, e passa-se a ter visibilidade as relações que se estabelecem entre as cidades vizinhas.

Ao analisar o caso do diferencial homem-mulher que pendulam por motivo escolaridade, pode-se observar que existe uma forte distinção de experiência, e, as mulheres são significativamente mais escolarizadas e informadas do que os homens.

Uma maioria desses indivíduos que pendulam residem em municípios tais como: Fortaleza, Caucaia, Maracanaú, Pacatuba, Crato e Juazeiro do Norte. E faz regularmente o trajeto casa/escola para municípios de Fortaleza, sobral, Juazeiro do Norte, Crato e Maracanaú, Quixadá e Limoeiro do Norte.

De modo geral, pode-se afirmar que no Ceará as mulheres jovens e solteiras apresentam um papel importante no processo de pendularidade em detrimento da participação dos homens, bem como níveis de escolaridade mais elevados em função, principalmente, de um acentuado desfavorecimento no mercado de trabalho, o que as impulsiona na busca por maior número de anos de estudo para uma melhor profissionalização. Além disso com a presença de políticas públicas voltadas para uma maior descentralização e ampliação das instituições de ensino os indivíduos percorrem distâncias cada vez menores para estudar, mais ainda sendo necessário sair do município de residência para aquisição de maiores instruções. Assim, a pendularidade, aqui, mostra efeitos importantes na estrutura social do Estado do Ceará que merecem maior atenção aos estudos populacionais.

\section{Referências}

ÂNTICO. C. Deslocamentos pendulares na Região metropolitana De São Paulo. São Paulo em Perspectiva. v. 19. n. 4. p. 110-120, 2005.

BAYLÃO, A. L. da S AS; CHETTINO, E. M. O. Inserção da Mulher no Mercado de Trabalho Brasileiro. In: XI Simpósio de excelência em gestão e tecnologia. 2014. Disponível em: <http://www.aedb.br/seget/arquivos/artigos14/20320175.pdf >. Acesso em: 18/03/2017.

BECKER, O. M. S. Deslocamentos Populacionais Pendulares em Áreas Ligadas À Indústria Petrolífera no Estado Do Rio de Janeiro, Brasil, 20001. Revista Geográfica de América Central, Número Especial EGAL. 2011 
BRITO. D. J. M.. Ensaios sobre deslocamento pendulares: uma análise para a região Metropolitana do Recife. Dissertação (Mestre). Programa de Pós-Graduação em Economia PPGE. João Pessoa. 2014. pp. 1-15

BRITO, Fausto; SOUZA, Joseane. Expansão urbana nas grandes metrópoles o significado das migrações intrametropolitanas e da mobilidade pendular na reprodução da pobreza. São Paulo em Perspectiva, v. 19, n. 4, p. 48-63, out./dez. 2005.

CUNHA, J. M. P.; JAKOB, A. A. E. Segregação socioespacial e inserção no mercado de trabalho na Região Metropolitana de Campinas. Revista Brasileira de Estudos

Populacionais, v. 27, n. 1, p. 115-139, jan./jun. 2010.

FREY, H. DOTA, E. M. O Censo De 2010 e as Primeiras Leituras Sobre a Mobilidade Espacial da População na Região Metropolitana de Campinas. Revista Mediações, v18.n1.p.226

INSTITUTO BRASILEIRO DE GEOGRAFIA E ESTATÍSTICA-IBGE. Censo Demográfico 2010. Rio de Janeiro: IBGE. 2010.

Estados. Disponível em: <http://www.ibge.gov.br/estadosat/perfil.php?sigla=ce>. Acesso em: 12/01/2017.

Migração e deslocamento, resultados da amostra, comentário dos resultados. Censo Demográfico 2000. Rio de Janeiro: IBGE, 2001. Disponível em:<http://biblioteca.ibge.gov.br/visualizacao/periodicos/88/cd_2000_migracao_deslocament o_amostra.pdf<Acesso em: 17/03/2017.

INSTITUTO DE PESQUISA E ESTRATÉGIA ECONÔMICA DO CEARÁ - IPECE. Ceará Mapas: Caracterização territorial. Disponível em:

<http://www2.ipece.ce.gov.br/atlas/capitulo1/11/111x.htm>. Acesso em: 12/01/2017.

Inserção feminina no mercado de trabalho do Ceará: avanço e persistência das diferenças entre gêneros. In: Enfoque Econômico, No 61, 2013. Disponível em:< http://www.ipece.ce.gov.br/enfoque-economico>. Acesso em: 17/03/2017.

INSTITUTO MAURO BORGES DE ESTATÍSTICAS E ESTUDOS SOCIOECONÔMICOS - IMB. Mobilidade Pendular da População em Goiás. 2012. Disponível em:〈http://www.imb.go.gov.br/down/mobilidade_pendular_da_populacao_em_goias.pdf $>$. Acesso em: 17/03/2017.

JARDIM, A. P. Reflexões sobre a mobilidade pendular. In: Luiz Antonio Pinto de Oliveira; Antônio Tadeu Ribeiro de Oliveira. (Org.). Reflexões sobre os Deslocamentos

Populacionais no Brasil. 1ed.Rio de Janeiro: Instituto Brasileiro de Geografia e Estatística IBGE, 2011, v. 01, p. 61- 73.

Mobilidade intrametropolitana: o caso do Rio de Janeiro. [s.l] Universidade Federal do Rio de Janeiro. 2001.

Movimentos pendulares: velhos e novos significados. In: V Encontro Nacional sobre Migrações. 2007. 
MARANDOLA, E. Jr. OJIMA, R. Pendularidade e vulnerabilidade na Região Metropolitana de Campinas: repercussões na estrutura e no habitar urbano. R. B. Estudos Urbanos e Regionais, v.16, n.2, p.185-204, 2014

MOURA, R; BRANCO, M. L. G. C.; FIRKOWSKI, O. C. DE F. Movimento pendular e Perspectivas de pesquisas em Aglomerados urbanos. São Paulo em Perspectiva, v. 19, n. 4, p. 121-133, 2005

MOREIRA, F. F et al. Mobilidade pendular entre as idades de Castanhal e Igarapé-Açu (PA) como alternativa para o ingresso no ensino superior. In: XIII Simpósio Brasileiro de Geografia Urbana - SIMPURB, Anais... Rio de Janeiro - RJ, 2013.

JARDIM, A. P. Reflexões sobre a mobilidade pendular. In: Luiz Antonio Pinto de Oliveira; Antônio Tadeu Ribeiro de Oliveira. (Org.). Reflexões sobre os Deslocamentos

Populacionais no Brasil. 1ed.Rio de Janeiro: Instituto Brasileiro de Geografia e Estatística IBGE, 2011, v. 01, p. 61- 73.

OJIMA, R.; SILVA, R. B. e PEREIRA, R. H. A mobilidade pendular na definição das cidades dormitório: caracterização sociodemográfica e novas territorialidades no contexto da urbanização brasileira. In: V Encontro Nacional sobre Migrações. Universidade Estadual de Campinas. Campinas, 2007.

OJIMA. R. O.; FUSCO. W. Nordeste do Brasil: interiorização do ensino superior e mobilidade pendular in: XX Encontro Nacional de Estudos Populacionais, Anais... Foz do Iguaçu/PR - Brasil, 2016.

OJIMA. R. O.; SILVA. R.B. da; PEREIRA. Rafael H. Moraes. A Mobilidade Pendular na Definição das Cidades-Dormitório: caracterização sociodemográfica e novas territorialidades no contexto da urbanização brasileira. In: V Encontro Nacional de Migrações. 2007.

OJIMA, R. Análise comparativa da dispersão urbana nas aglomerações urbanas brasileiras: elementos teóricos e metodológicos para o planejamento urbano e ambiental. 2007. Tese (Doutorado em Demografia) - Instituto de Filosofia e Ciências Humanas, Universidade Estadual de Campinas, Campinas.

PESSOA. Z. S.. Uma análise do movimento pendular na definição da espacialidade territorial e social na RMNATAL. In: XI Encontro Nacional da Associação Nacional de Pós-Graduação e Pesquisa em Planejamento Urbano e Regional - ANPUR. Anais... Salvador. Bahia. 2005.

PIÇANGO, F.Juventude e acesso ao ensino superior: novo hiato de gênero. In: ITABORAI.N.R; RICOLDI, A. M. (Org.). Até onde caminhou a Revolução de gênero no Brasil: implicações demográficas e questões sociais. Belo Horizonte, MG: ABEP, 2016. p.117-132.

SILVA, R. B. Mobilidade Pendular, população e vulnerabilidade socioambiental na Região Metropolitana da Baixada Santista: um olhar sobre Cubatão. In: XVI Encontro Nacional de Estudos Populacionais. Anais... Caxambu- MG - Brasil, 2008. 
SISTEMA DE PESQUISA DE EMPREGO E DESEMPREGO - PED. Inserção das mulheres de ensino superior no mercado de trabalho. 2011. Disponível

em: <https://www.dieese.org.br/analiseped/2011/2011pedmulherfor.pdf >. Acesso em:17/03/2017.

STAMM, C.; STADUTO, J. A. R. Movimentos pendulares das cidades interioranas de porte médio de Cascavel e Toledo, no Paraná. Revista brasileira de Estudos da População, São Paulo, v. 25, n. 1, p. 131-149, jan./jun. 2008. 